detection in acute myeloid leukemia: data from the hovon/sakk aml 42a study. J Clin Oncol 2013; 31: 3889-3897.

14 Silva FP, Swagemakers SM, Erpelinck-Verschueren C, Wouters BJ, Delwel R, Vrieling $\mathrm{H}$ et al. Gene expression profiling of minimally differentiated acute myeloid leukemia: M0 is a distinct entity subdivided by runx 1 mutation status. Blood 2009; 114: 3001-3007.
15 Shlush LI, Zandi S, Mitchell A, Chen WC, Brandwein JM, Gupta V et al. Identification of pre-leukaemic haematopoietic stem cells in acute leukaemia. Nature 2014; 506: 328-333.

$16 \mathrm{Xu} \mathrm{X,} \mathrm{Wells} \mathrm{A,} \mathrm{Padilla} \mathrm{MT,} \mathrm{Kato} \mathrm{K,} \mathrm{Kim} \mathrm{KC,} \mathrm{Lin} \mathrm{Y.} \mathrm{A} \mathrm{signaling} \mathrm{pathway} \mathrm{consisting} \mathrm{of}$ miR-551b, catalase and MUC1 contributes to acquired apoptosis resistance and chemoresistance. Carcinogenesis 2014; 35: 2457-2466.

Supplementary Information accompanies this paper on the Leukemia website (http://www.nature.com/leu)

\title{
OPEN
}

\section{Distinct molecular abnormalities underlie unique clinical features of essential thrombocythemia in children}

\section{Leukemia (2016) 30, 746-749; doi:10.1038/leu.2015.167}

Essential thrombocythemia (ET) is rare in children, with an annual incidence of $\sim 100$-fold lower than that in adults. ${ }^{1}$ The rarity of the disease in children makes the clinical course and pathogenesis of childhood ET far less clear. It is reported that clonal markers are much less common in children with ET (25.8\%) than that in adult cases (80-90\%). ${ }^{2-4}$ Except for JAK2 V617F, and MPL and calreticulin (CALR) mutations, no other mutations have yet been reported in childhood ET. ${ }^{2,5-8}$ More molecular markers are needed to distinguish clonal from reactive thrombocytosis in children. The present study investigated the JAK2 V617F, and MPL and CALR mutations in a large cohort of children with ET. We conducted the first study to analyze the molecular profiles by targeted nextgeneration sequencing and to investigate the JAK2 46/1 haplotype in childhood ET.

Sixty-three children diagnosed with sporadic ET according to the 2008 World Health Organization criteria were enrolled.9 ${ }^{9}$ Bone marrow histology was consistent with a diagnosis of ET in all cases. The molecular patterns were evaluated before any cytoreductive drug use. The JAK2 V617F, and MPL and CALR mutations were investigated as previously reported. ${ }^{4}$ The JAK2 46/1 haplotype (rs12340895) was assessed by Sanger sequencing. Fifty-five genes (Supplementary Table 1) associated with myeloid malignancies were analyzed by targeted sequencing in 25 children. Polymorphisms in existing database were excluded. Mutations were validated by Sanger sequencing (Supplementary Table 2). Each true-positive mutation was further investigated in 100 normal controls. Germline DNA was used to identify somatic mutations. Detailed information is shown in the Supplementary Methods.

The median age was 11 years (range, 3-14 years). Different from adult $\mathrm{ET}^{10}$ a male preponderance was observed among childhood patients, with a male/female ratio of $1.5(38 / 25)$. Compared with the adult patients that we previously reported, ${ }^{10}$ childhood ET had higher platelet counts (median $1224 \times 10^{9}$ vs $900 \times 10^{9} /$; $P<0.001$ ), lower hemoglobin level (median 127 vs $137 \mathrm{~g} /$; $P<0.001$ ) and comparable white blood cell counts (median $10.6 \times 10^{9}$ vs $9.9 \times 10^{9} /$; $P=0.312$ ). It indicates a pronounced and isolated megakaryocyte proliferation in child patients. The JAK2 V617F mutation was found in 14 children (22.2\%), with a median allele frequency of $22 \%$ (range, 10-31\%). In 49 patients among whom CALR and MPL mutations were investigated, only an 11-year-old girl harbored a CALR mutation (52-bp deletion), and none had MPL mutations. The molecular markers were much less common than that in adult patients. ${ }^{3,4}$ In adult patients, compared with patients with wild-type JAK2, V617F-mutated patients display higher white blood cell counts, higher hemoglobin level but lower platelet counts. ${ }^{4}$ However, there were no differences in blood cell counts between children with and without the JAK2 V617F mutation (Supplementary Table 3).

Three children (4.8\%) displayed major thrombosis, and two of them were V617F-mutated. Microvascular disturbances were more common $(n=30,47.6 \%)$ than that in Caucasian children $(30.3 \%){ }^{2}$ Headache was the most frequent symptom $(n=22,34.9 \%)$. The incidence of headache was significantly related to platelet counts at diagnosis $(P=0.013)$. Headache was relieved by antiplatelet and/or cytoreductive therapy in 17 children (77.3\%), providing important evidence for the central role of platelets in the etiology of headache. No major bleeding events were observed. The rate of minor bleeding episodes was $14.3 \%(n=9)$, similar to that in the Caucasian children $(9.0 \%)^{2}$ The risk of minor bleeding events was not related to platelet counts $(P=0.126)$ or JAK2 V617F mutation $(P=0.194))$. Two children (3.2\%) evolved to myelofibrosis after 20 and 7 years of follow-up, respectively. One harbored the JAK2 V617F mutation and received 1 year of intermittent hydroxyurea treatment before transformation. The other one did not have JAK2 V617F or CALR mutations, and received 2 months of interferonalpha and then 5 years of hydroxyurea before transformation.

Targeted sequencing was performed in 25 children (Supplementary Table 4). The average depth (median, 349-fold) and coverage (median 99.6\%) of the target regions were excellent (Supplementary Figure 1). A total of 135 single-nucleotide variants were identified (Supplementary Table 5). A detailed filtration pipeline was developed to select mutations that might be related to tumorigenesis (Supplementary Figure 2). After filtration, eighteen types of somatic mutations (single nucleotide variants and indels) involving 13 genes and 7 germline mutations involving 7 genes were selected (Supplementary Table 6). Somatic mutations were present in 14 children (56.0\%), with a median allele frequency of $33.6 \%$ (range, 18.8-60.4\%). Other than JAK2 V617F $(n=6,24.0 \%)$, the most frequently observed somatic mutations were ASXL1 mutations ( $n=4,16.0 \%)$ (Figure 1a). In 18 children with wild-type JAK2 and CALR, seven (38.9\%) harbored somatic mutations that were previously undocumented in childhood ET. Six $(24.0 \%)$ children harbored two or more somatic mutations (Figure 1b).

The molecular profiles were different between childhood and adult ET patients. On the one hand, mutations that were commonly involved in adult ET were not found in childhood ET. In adult patients, other than JAK2, CALR and MPL, mutated genes most commonly include TET2 (4\%-11\%) and DNMT3A (1-5\%). ${ }^{11}$ However, none of the children had TET2 or DNMT3A mutations. On 
a

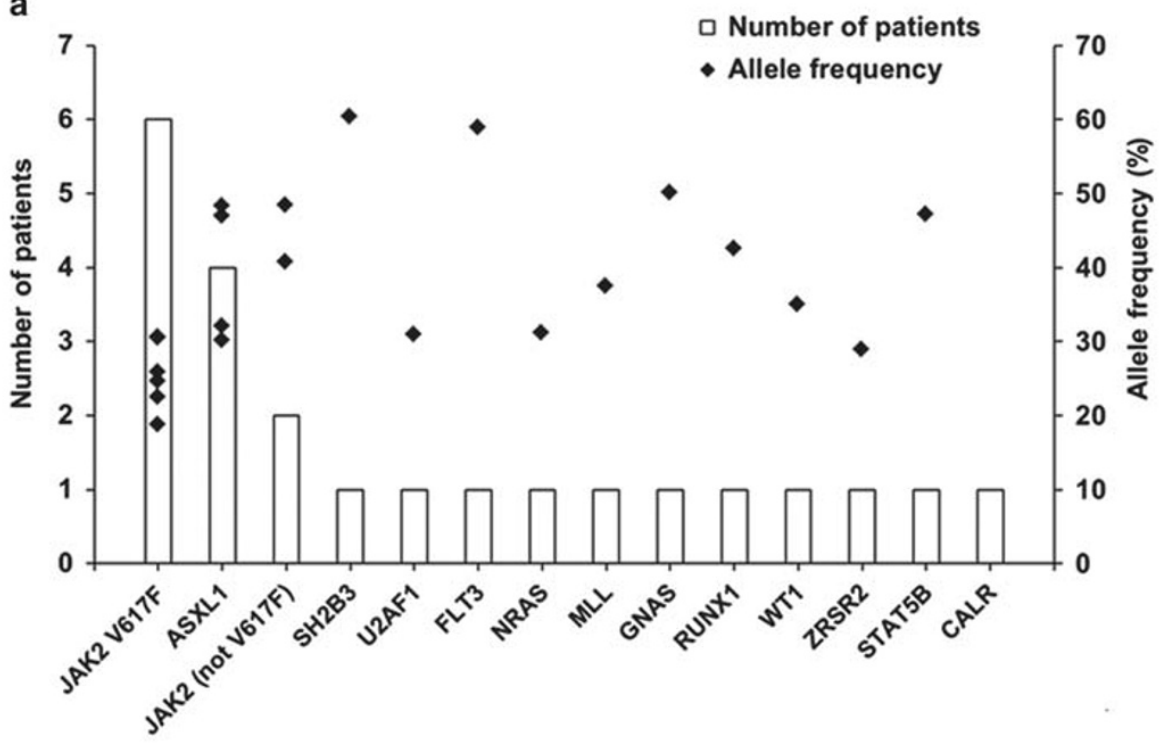

b

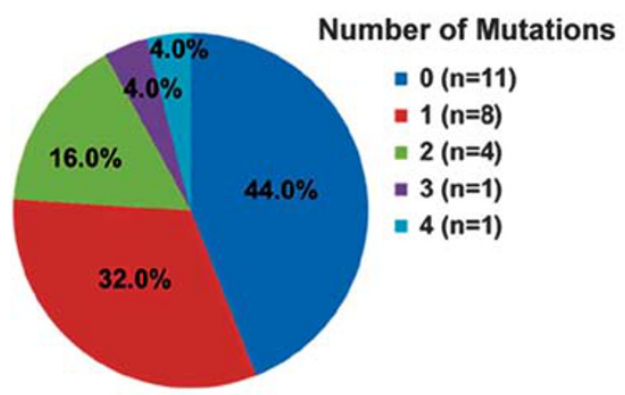

C

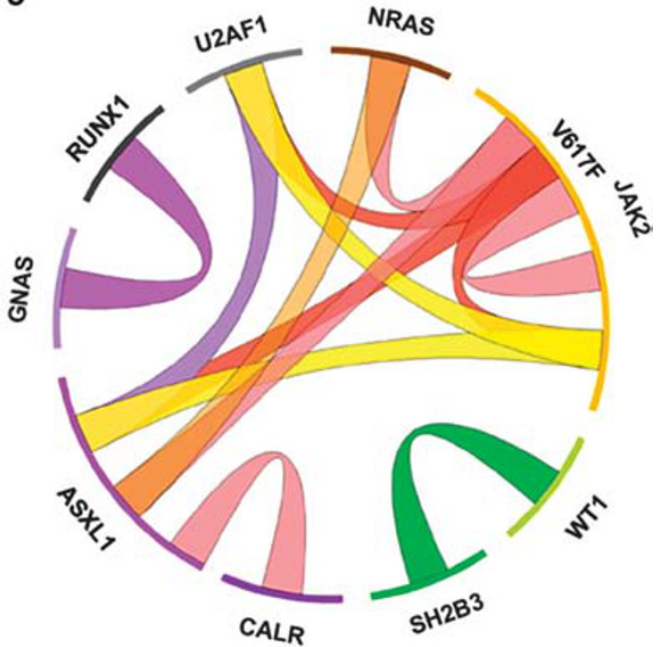

Figure 1. Frequency and distribution of somatic mutations in children with essential thrombocythemia. (a) Number of patients and allele frequency of each mutated gene. (b) Number of patients with different number of somatic mutations. (c) Co-occurrence of the somatic mutations in the same individual.

the other hand, the newly identified mutations in childhood ET (that is, mutations in the gene NRAS, MLL, U2AF1, ZRSR2, GNAS, FLT3, RUNX1 and WT1) were rarely seen in adult ET but were recurrent in myelodysplastic syndrome, primary myelofibrosis or blast phase myeloproliferative neoplasms. ${ }^{11-13}$ The rate of the ASXL1 mutations was much higher (16\%) than that in adult ET $(2-5 \%) .{ }^{11}$ The discovery of recurrent mutations indicates that targeted sequencing can be used to distinguish clonal from reactive thrombocytosis in children.

By analyzing co-occurrence of the somatic mutations (Figure 1c), we revealed a genetic complexity in childhood ET. Lundberg et al. ${ }^{14}$ reported that in 60 adults with somatic mutations, $17(28.3 \%)$ had two or more mutations. In our study, about half of the children with somatic mutations $(6 / 14,42.9 \%)$ had more than one mutation. The higher rate of co-occurrence of rare somatic mutations in childhood ET suggests that children with ET might have a more complex and unstable genetic composition than adult patients have. Acquisition and accumulation of the somatic mutations in early life might be the main reason for the early onset of ET in children. Mutual exclusivity was observed in gene pairs with similar biological function, such as genes both involved in the JAK-STAT pathway. However, co-occurrence of mutations in different alleles of JAK2 (that is, JAK2 V617F and JAK2 I354T; JAK2 V617F and JAK2 G127D) was observed in two children, indicating that the JAK2 gene might be more sensitive to the genetic instability. This might be one of the reasons why JAK2 was most commonly affected in ET. The definite existence of genetic instability needs more evidence.

The JAK2 46/1 haplotype was assessed in 49 children. The number of children with CC, CG and GG genotypes was 17 $(34.7 \%), 31(63.3 \%)$ and $1(2.0 \%)$, respectively. The frequency of the JAK2 46/1 haplotype (33.7\%) was significantly higher than that in normal Chinese population $(21.9 \% ; P=0.013) .{ }^{15}$ It suggests that inherited predisposition may also exist in childhood patients.

Clusters of mutations based on allele frequencies distinguish founding clones from subclones. ${ }^{12}$ Figure 2 shows diverse clonal hierarchies in two representative children. The germline IDH1 mutation in patient E1 and the JAK2 46/1 haplotype in patient E3 reflected the inherited genetic background. In patient E1, three variants (that is, NRAS, ASXL1 and JAK2 V617F) with similar allele frequencies $(\sim 30 \%)$ might exist in the same founding clone or in separate founding clones (Figures $2 \mathrm{a}$ and b). Different from 

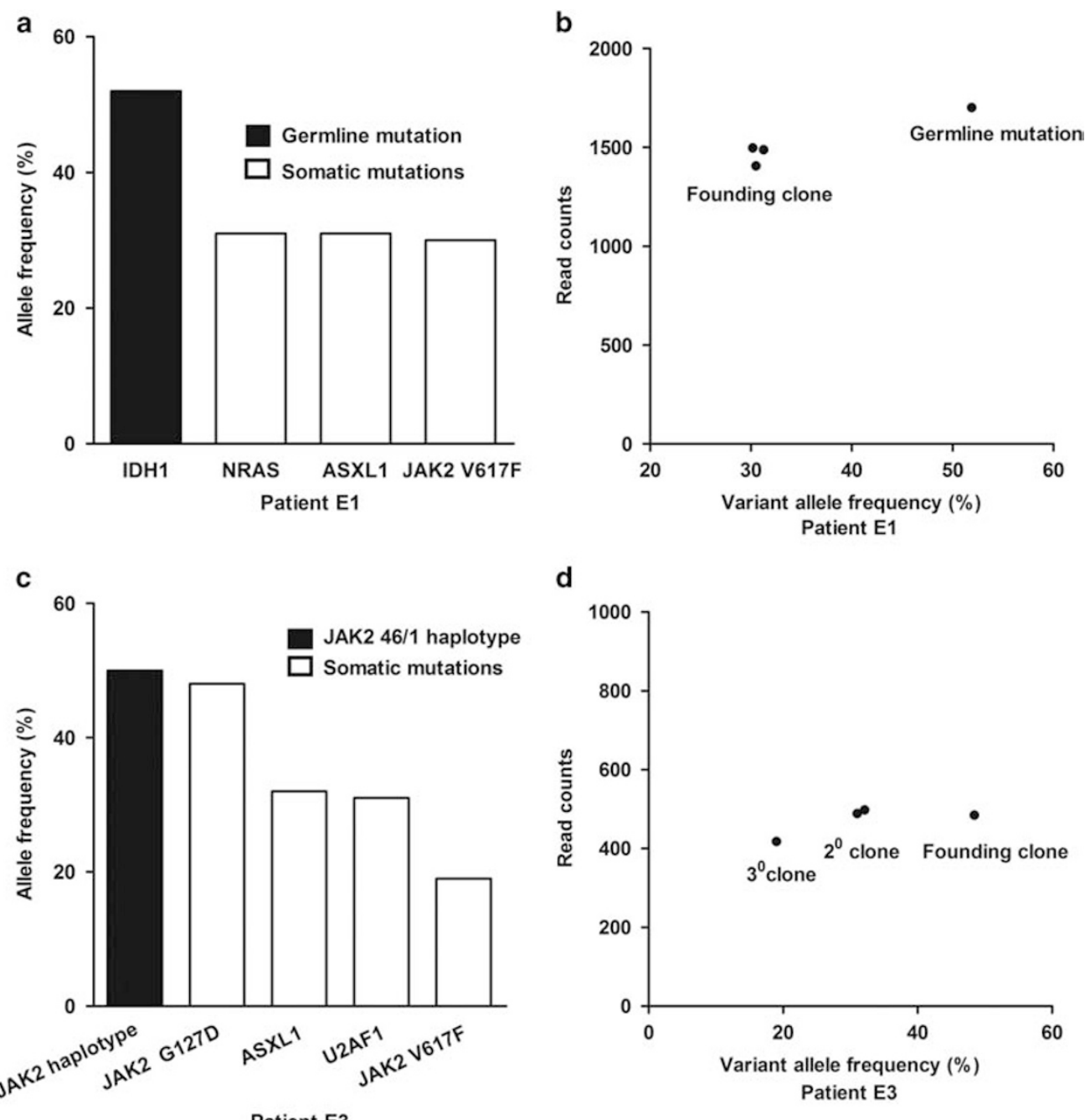

Figure 2. Clonality assessment in two representative cases of essential thrombocythemia. (a) Allele frequencies of mutations in patient E1. (b) Clusters of variants identify the founding clone in patient E1. (c) Allele frequencies of mutations in patient E3. (d) Clusters of variants identify the founding clone and subclones in patient E3. In (b) and (d), the allele frequency is plotted versus the total number of sequencing reads covering the corresponding mutated nucleotide.

patient E1, patient E3 had the founding clone (that is, JAK2 G127D), first-level subclones (that is, ASXL1 and U2AF1), and second-level subclone (that is, JAK2 V617F) (Figures 2c and d). Since the heterozygous JAK2 G127D mutation (allele frequency, 48.5\%) was present in almost all cells, JAK2 V617F (allele frequency, $19.0 \%)$ was not the earliest genetic alteration. Similarly, the CALR mutation was also not the initial abnormality, which could be speculated from the co-occurrence of the heterozygous ASXL1 M1096L mutation (allele frequency, 48.4\%) and the CALR mutation (allele frequency, 21.1\%) in the same individual.

Concerning gender, age and blood cell counts, no differences were found between mutated and non-mutated children, except for white blood cell counts $(P=0.025)$. The only child with thrombosis among the 25 children had a single ASXL1 mutation. The types of antiplatelet and cytoreductive treatment between mutated and non-mutated children were similar (Supplementary Table 4).

In conclusion, the molecular profiles are different between childhood and adult ET patients, and the genetic composition in childhood ET may be more complex than that in adults. The difference in clinical and hematological characteristics between childhood and adult ET may be due to the different molecular profiles underlying the two entities. Additional molecular markers are found by targeted sequencing to identify children with a clonal blood disorder. After JAK2 V617F, the most frequently observed somatic mutations are ASXL1 mutations. The JAK2 V617F or CALR mutations may not be the initial abnormalities in some children. Whole genome sequencing would help to reveal initial molecular events.

\section{CONFLICT OF INTEREST}

The authors declare no conflict of interest.

\section{ACKNOWLEDGEMENTS}

This study was supported by 863 projects of the Ministry of Science and Technology of China (2012AA02A211) (LZ), and National Natural Science Foundation of China (81270595 and 81470302) (LZ). English editing service was provided by Editage (http://www.editage.cn/).

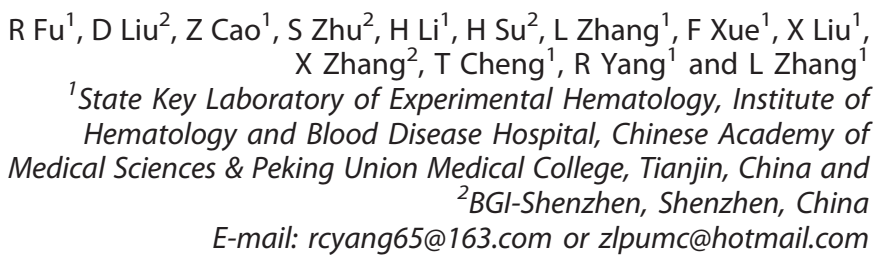




\section{REFERENCES}

1 Hasle H. Incidence of essential thrombocythaemia in children. Br J Haematol 2000; 110: 751.

2 Randi ML, Geranio G, Bertozzi I, Micalizzi C, Ramenghi U, Tucci F et al. Are all cases of paediatric essential thrombocythaemia really myeloproliferative neoplasms? Analysis of a large cohort. Br J Haematol 2015; 169: 584-589.

3 Nangalia J, Massie CE, Baxter EJ, Nice FL, Gundem G, Wedge DC et al. Somatic CALR mutations in myeloproliferative neoplasms with nonmutated JAK2. N Engl J Med 2013; 369: 2391-2405.

4 Fu R, Xuan M, Zhou Y, Sun T, Bai J, Cao Z et al. Analysis of calreticulin mutations in Chinese patients with essential thrombocythemia: clinical implications in diagnosis, prognosis and treatment. Leukemia 2014; 28: 1912-1914.

5 Giona F, Teofili L, Capodimonti S, Laurino M, Martini M, Marzella D et al. CALR mutations in patients with essential thrombocythemia diagnosed in childhood and adolescence. Blood 2014; 123: 3677-3679.

6 Langabeer SE, Haslam K, McMahon C. CALR mutations are rare in childhood essential thrombocythemia. Pediatr Blood Cancer 2014; 61: 1523.

7 Kucine N, Chastain KM, Mahler MB, Bussel JB. Primary thrombocytosis in children. Haematologica 2014; 99: 620-628.

8 Fu R, Zhang L, Yang R. Paediatric essential thrombocythaemia: clinical and molecular features, diagnosis and treatment. Br J Haematol 2013; 163: 295-302.

9 Tefferi A, Vardiman JW. Classification and diagnosis of myeloproliferative neoplasms: the 2008 World Health Organization criteria and point-of-care diagnostic algorithms. Leukemia 2008; 22: 14-22.

10 Fu R, Xuan M, Lv C, Zhang L, Li H, Zhang X et al. External validation and clinical evaluation of the International Prognostic Score of Thrombosis for Essential
Thrombocythemia (IPSET-thrombosis) in a large cohort of Chinese patients. Eur J Haematol 2014; 92: 502-509.

11 Tefferi A. Novel mutations and their functional and clinical relevance in myeloproliferative neoplasms: JAK2, MPL, TET2, ASXL1, CBL, IDH and IKZF1. Leukemia 2010; 24: 1128-1138.

12 Papaemmanuil E, Gerstung M, Malcovati L, Tauro S, Gundem G, Van Loo P et al. Clinical and biological implications of driver mutations in myelodysplastic syndromes. Blood 2013; 122: 3616-3627.

13 Bejar R, Stevenson K, Abdel-Wahab O, Galili N, Nilsson B, Garcia-Manero G et al. Clinical effect of point mutations in myelodysplastic syndromes. $N$ Engl J Med 2011; 364: 2496-2506.

14 Lundberg P, Karow A, Nienhold R, Looser R, Hao-Shen H, Nissen I et al. Clonal evolution and clinical correlates of somatic mutations in myeloproliferative neoplasms. Blood 2014; 123: 2220-2228.

15 Zhang X, Hu T, Wu Z, Kang Z, Liu W, Guan M. The JAK2 46/1 haplotype is a risk factor for myeloproliferative neoplasms in Chinese patients. Int J Hematol 2012; 96: 611-616.

\footnotetext{
This work is licensed under a Creative Commons AttributionNonCommercial-NoDerivs 4.0 International License. The images or By No ND license, unless indicated otherwise in the credit line; if the material is not included under the Creative Commons license, users will need to obtain permission from the license holder to reproduce the material. To view a copy of this license, visit http:// creativecommons.org/licenses/by-nc-nd/4.0/
}

\section{Gene dosage reductions of Trf1 and/or Tin2 induce telomere DNA damage and lymphoma formation in aging mice}

Leukemia (2016) 30, 749-753; doi:10.1038/leu.2015.173

Telomeres are essential structures that cap the end of chromosomes, which is required for maintenance of chromosomal stability, cell viability and the capacity of cells to proliferate. A complex of specific telomere-binding proteins (TRF1, TRF2, POT1, TIN2, TPP1 and RAP1), also known as the Shelterin complex, is essential for telomere capping by assisting the formation of tertiary telomeric structures. ${ }^{1}$ Gene mutations in components of the Shelterin complex ( $h$ TIN2, hPOT1 and hTPP1) lead to bone marrow failure and cancer formation in human genetic diseases including dyskeratosis congenita (DC), which is caused by Tin2 mutation in $20 \%$ of the cases. ${ }^{2,3}$ All known TIN2 mutations are heterozygous, autosomal-dominant and patients normally show extremely short telomeres. In addition, mutations in the telomere binding protein POT1 were shown to lead to lymphocytic leukaemia formation. ${ }^{4}$ Aside from genetic diseases, a variety of studies reported reduced expression of telomere-binding proteins in human cancers compared with non-cancerous tissue suggesting that downregulation of the expression of telomere-binding proteins may also contribute to carcinogenesis in somatic cells and tissues. ${ }^{5,6}$ It was shown that Epstein-Barr virus-encoded LMP1 and Epstein-Barr virus-infection itself induce the downregulation of TRF1, TRF2 and POT1 at the transcriptional and translational level resulting in complex chromosomal aberrations, alternative lengthening of telomeres and the induction of Hodgkin's lymphoma. ${ }^{7,8}$

The causal relation between gene dosage reductions of telomere binding protein and the development of cancer and tissue aging remains elusive. Mouse knockout studies revealed that homozygous deletions of Tin2 or Trf1 lead to early embryonic lethality. ${ }^{9,10}$ The conditional homozygous deletion of Trf1 was shown to provoke severe defects in tissue maintenance ${ }^{11}$ and in combination with homozygous p53 deletion led to cancer formation in skin. ${ }^{12}$ However, these models did not address the question of whether moderate reductions in the gene dose of telomere-binding proteins contribute to tissue aging and/or carcinogenesis. To address this question we followed aging cohorts of mice carrying heterozygous deletion of Trf1 and/or Tin2 in comparison with wild-type mice. ${ }^{9,10}$

Heterozygous Trf $^{+/}{ }^{+-}$Tin2 $^{+/-}$knockout mice showed a $40-50 \%$ reduction in the mRNA expression level of Trf1 and Tin2, but had no effect on the mRNA expression profile of other telomerebinding proteins (Figures 1a and b, Supplementary Figures 1a and d). Protein analysis of whole-spleen extracts revealed an $\sim 50 \%$ reduced Tin2 expression in Tin2 $2^{+/-}$mice compared with $\operatorname{Tin} 2^{+/+}$ mice (Figure 1c, Supplementary Figure 1e). Trusty antibodies for detection of endogenous Trf1 protein in tissues are still lacking. To monitor the Trf1 protein amounts in Trf1 heterozygous mice, a Trf1 hemagglutinin ( $\mathrm{HA}$ )-tag knockin mouse line was generated carrying the HA-tag at the $\mathrm{N}$-terminus of the endogenous Trf1 gene locus. Opposed to the homozygous Trf1 knockout mouse, homozygous HA-Trf1 knockin mice (Trf ${ }^{\mathrm{ki} / \mathrm{ki}}$ ) are viable, do not exhibit an overt organismal phenotype and show normal telomere structure indicating that the HA-tag did not interfere with Trf1 function. Trf1 protein from the knockin mice was quantitatively immunoprecipitated with an anti-HA antibody using equally concentrated lysates. Heterozygous Trf1 deletion led to a reduction in Trf1 protein amounts, whereas the heterozygous deletion of Tin2 had no significant impact on Trf1 protein levels 